\title{
Química e Saúde Pública: Elementos da História de uma relação fundamental
}

\author{
Chemistry and Public Health: \\ Elements of the History of a Fundamental Relationship
}

\author{
Sérgio P. J. Rodrigues, Universidade de Coimbra, CQC Departamento de Química \\ (spjrodrigues@ci.uc.pt)
}

\begin{abstract}
Resumo: A química teve, a partir do final do século XVIII, um papel central, que se mantém na atualidade, na saúde pública e no bem-estar da humanidade. A sua maior contribuição é na segurança alimentar, que contribuiu para salvar as vidas de cerca de um terço da população humana. O tratamento da água de consumo com cloro contribuiu para salvar da morte prematura quase duzentos milhões de pessoas. Doenças como a febre tifoide, que mataram reis, como D. Pedro V, e a cólera, são hoje essencialmente memórias. Os antibióticos contribuíram para quase eliminar a sífilis e outras infeções. A tuberculose e a malária quase desapareceram na Europa, em boa parte devido à química. Também a assepsia, a anestesia e a segurança das transfusões de sangue e das vacinas devem muito a contribuições químicas. Atualmente, estamos a viver uma situação de pandemia global, mas devido, também à ciência, podemos enfrentar os problemas com esperança. O principal objetivo do presente trabalho é fazer uma revisão histórica das contribuições químicas para a saúde pública e bem-estar, algo que parecendo trivial, raramente é compreendido em toda a sua extensão.
\end{abstract}

Palavras-chave: Contribuições Químicas, Saúde Pública, Alimentação, Higiene, Medicamentos

\begin{abstract}
Chemistry has, since the end of the XVIIIth century, a central role, maintained nowadays, in the public health and well-being of humankind. Its major contribution is on food safety, which contributed to saving the lives of around a third of the human population. The treatment of tap water with chlorine contributed to saving from premature death around two hundred persons. Diseases such as typhoid fever, which killed kings, as D. Pedro V, and cholera, are now mainly memories. The antibiotics contributed to nearly eliminating syphilis and other infections. Tuberculosis and malaria almost disappeared in Europe, due in great part to chemistry. Also, asepsis, anesthesia, and the safety of blood transfusions and vaccines owe much to chemical contributions. Nowadays we are passing through a global pandemic crisis, but due to science, we can face the problems with hope. The main aim of the present work is to review the history of chemical contributions to public health and well-being, a subject that appears trivial and is seldom understood to all their extent.
\end{abstract}

Keywords: Chemical Contributions, Public Health, Food, Hygiene, Medicines 


\section{Introdução}

A química ${ }^{1}$ tem contribuído desde o seu aparecimento para a saúde pública e o bem-estar de várias maneiras. São basicamente três as formas de contribuição que salvaram mais vidas humanas. ${ }^{2}$ A alimentação é a principal, depois a higiene, e, finalmente, os tratamentos médicos e medicamentos. Na segurança alimentar, esta contribuição envolve a produção necessária para evitar a fome da população e o controle de qualidade do que é comido. No que respeita à higiene, esta ciência apresenta muitas contribuições, desde os detergentes e desinfetantes até à distribuição e tratamento de água e saneamento. Finalmente, de forma aparentemente mais direta, através da descoberta, desenvolvimento e produção de medicamentos e desenvolvimento de tratamentos médicos, a química contribui também para a saúde pública e bem-estar. Entretanto, a resolução de muitos destes problemas acarretou outros, que, de novo, a química também se tem esforçado para resolver. Muitas destas contribuições, assim como os seus resultados, são tão óbvias que acabamos, muitas vezes, por as considerar triviais e garantidas, mas demoraram muito tempo a ser desenvolvidas e, sobretudo, houve tempos em que não existiram.

Deve notar-se que os apectos científicas comuns raramente aparecem nas notícias, contrariamente aos problemas resultantes destas, que têm quase sempre destaque. Ainda bem, pois um dos objetivos da ciência é resolver os problemas que vão surgindo. Mas os novos problemas não devem fazer-nos esquecer as resoluções que foram conseguidas para problemas antigos.

O diálogo entre o pessimismo e o otimismo exagerado foi explorado por vários autores (e.g., Emsley, 2010; Keinam, 2013; Rodrigues, 2014, 2016a, 2016b; Pinker, 2018; Rosling, 2018, 2021) e continua a ser discutido. O objetivo do presente trabalho é essencialmente histórico, mas a pandemia de COVID-19 já se desenvolve há tempo suficiente para fornecer informação relevante para este trabalho.

Por um lado, a sociedade habituou-se, e espera, um nível de segurança muito alto. Mas por outro lado, e também devido às expectativas anteriores, as medidas

1 Esta ciência tem várias facetas, tanto teóricas como utilitárias. Em termos teóricos, procura explicar e agir sobre a matéria com base nas propriedades dos átomos, moléculas e iões que a compõem. Na sua ação sobre a matéria, tem uma grande componente utilitária, na qual se incluem a descoberta, o desenvolvimento e a produção de materiais e o domínio de processos, tanto naturais como artificiais. A química não procura só aumentar o nosso conhecimento sobre a Natureza mas também aumentar essa própria Natureza (Laszlo, 1996). Esta ciência não está só, obviamente, estando presente nas engenharias, na farmácia, na medicina e noutras áreas do conhecimento.

2 Entende-se "salvar vidas humanas" como o ato de contribuir de alguma forma para evitar mortes prematuras. 
que têm sido tomadas têm tido um impacto enorme na sociedade. São inúmeras as diretivas oficiais sobre o assunto e seria quase impossível fazer aqui um resumo. São exemplos, aquelas que são publicitadas pela Organização Médica Mundial (WHO, 2021a) das Nações Unidas (NU) e pela Agência Europeia do medicamento (Ema, 2021a) da União Europeia (UE). O número de vítimas mortais já é significativo (5,3 milhões, WHO 2021b; Worldometer, 2021b; Ritchie et al., 2021, ver também Cinar \& Ekinci, 2022), mas a mortalidade continua baixa (1.9\%, estimativa com base nos infetados e mortos registados). Mesmo no cenário de toda a população do mundo apanhar COVID-19, mantendo a mesma mortalidade, chegaríamos a números que, sendo considerados inaceitáveis (cerca de 150 milhões de vítimas, estimativa realizada a partir da mortalidade anterior e da população mundial, Worldometer, 2021a), são inferiores aos das piores pandemias da história da humanidade. Claro que há muitos motivos de preocupação, nomeadamente com as mutações do vírus, com o tempo de validade das vacinas, com o crescimento da pandemia e com a reinfeção (Wang, Kaperak \& Sato, 2021), mas há também indicações positivas (Christensen, 2021). Tal começou desde logo com o desenvolvimento muito rápido de vacinas. Isto foi devido à grande preparação da comunidade científica e a desenvolvimentos anteriores. Além disso, todo este trabalho tem sido analisado cuidadosamente, procurando aprender-se com o passado e a atualidade (o número de referências e revisões é muito grande - cito só como exemplo Ansah et al. 2021). E, depois, há que considerar os avanços que a epidemia acabou por originar em muitas áreas (mais uma vez, o número de trabalhos disponíveis é imenso. São aqui citados apenas alguns artigos de revisão, que já estando disponíveis, têm datas de publicação de 2022 e não tratam diretamente da pandemia, mas de assuntos relacionados, Felix et al. 2022; Kumar et al., 2022: Li et al., 2022; Porto \& Porto, 2022).

A Food and Drug Admistration (FDA, 2021b) aprovou o primeiro medicamento para uso oral em situações de emergência a 22 de dezembro de 2021. Este medicamento ainda não tinha sido aprovado na UE, mas pode também ser usado na Europa em situações de emergência (Ema, 2021c). Paralelamente, estão já disponíveis auto-testes de antigénio, os quais foram rapidamente desenvolvidos. Os testes de reação em cadeia de polimerase (PCR) são de enorme sensibilidade e foram rapidamente adaptados para a deteção do coronavirus e das suas mutações. Tudo isto tem sido desenvolvido a partir de conhecimentos e materiais anteriores. E, poder-se-ia pensar que estes desenvolvimentos pouco tinham a ver com a química, o que é errado. Desde logo os materiais de que são 
feitos. No caso dos auto-testes, o uso de nanopartículas de ouro, nitrocelulose e soluções tampão, etc. (Moabelo el al. 2021). Entretanto, o conhecimento, em particular o conhecimento científico, tem sido exponencial e cumulativo, e é, por isso, natural que o ritmo da realização de novas descobertas e invenções vá acelerando com o tempo. Mas isso não pode fazer-nos esquecer a história destes desenvolvimentos e invenções.

\section{Doenças e História: olhando para os seus efeitos nos privilegiados}

Várias doenças que quase desapareceram, e condições que são hoje triviais, vitimaram monarcas e imperadores. Na Figura 1 podemos encontrar uma montagem de autor desconhecido da família real portuguesa cerca de 1850 na qual foram acrescentadas as idades da morte e outras informações consideradas relevantes. Desde logo, notamos a idade da morte prematura de várias das pessoas envolvidas. Uma delas é a jovem rainha D. Estefânia que morreu de difteria. O rei D. Pedro V e vários dos irmãos e tios morreram de febre tifoide, na sequência de uma caçada em que beberam de uma fonte inquinada. A mãe, D. Maria II, tinha muito partos, sendo estes perigosos, acabando de morrer num deles. É também notória a mortalidade infantil e os nascidos mortos (no canto direito acima são apresentados os nados-mortos de D. Maria II). Entretanto, as pessoas que conseguiam sobreviver, podiam durar muito mais tempo, mas com uma existência cheia de dores e sofrimento. Baseado na família real portuguesa e meados do século XIX, podemos indicar imediatamente o que faltava nessa época: o tratamento da água de consumo, as vacinas, a segurança e higiene no parto, os anticoncecionais, os antibióticos, entre muitas outras coisas.

Os monarcas, e as pessoas que não eram da aristocracia, podiam também ser felizes, claro, mas não devemos esquecer estas ausências, de que hoje quase nem damos conta, de tal forma fazem parte da nossa vida (Landes, 2001, pp XVII$\mathrm{XX)}$. E, já na altura, não se dava conta desta ausência. $\mathrm{O}$ povo não aceitou a explicação da morte do monarca português, que atribuiu a veneno, e houve descontentamento público. Foi assim, realizada uma autópsia pública, conduzida pelos melhores médicos e químicos do país, a um dos príncipes, D. João. Esta foi publicada no Diário de Lisboa (1862), que era o diário oficial do governo, não tendo sido detetado qualquer veneno, confirmando a junta médica que foi a febre tifoide que vitimou o monarca. 


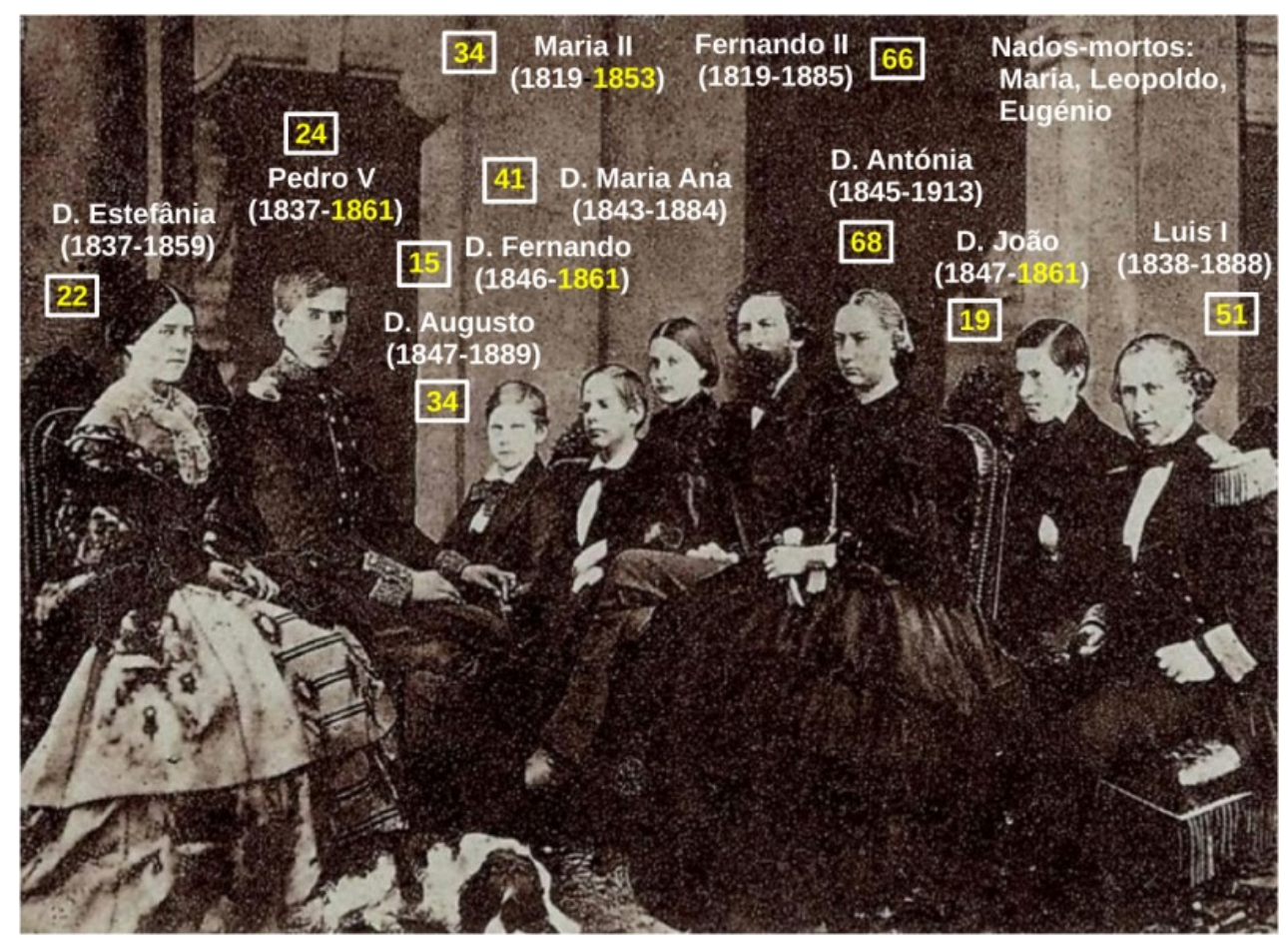

Figura 1: Família real portuguesa em 1858/59 (fotomontagem de autor desconhecido). Nesta figura podemos encontrar as datas de nascimento e de morte e tempo de vida (em anos). Foram também incluídos os nados-mortos da rainha D. Maria II.

Antes do século XIX, houve monarcas vitimados com peste (Mattoso, 2020, p. 191) e com lepra. Na Idade Média, a mortalidade infantil postulava-se muito alta, por as condições de higiene serem muito deficientes. A idade média de casamento das mulheres era de 15 anos (16 anos mais tarde) e a esperança de vida, depois dos 10 anos, de cerca de 40 anos (Mattoso, 2015, p. 560). A tuberculose, que começará a tornar-se importante no século XIX, é bastante particular, pois, embora tivesse sido muito romantizada, era uma doença que as más condições sanitárias e de alimentação agravava (Vieira, 2015). Entretanto, tivemos banqueiros e pessoas poderosas vitimadas com infeções (Landes, 2001, p. XVII) e depois artistas e pessoas famosas vitimadas de SIDA. É verdade que atualmente tivemos banqueiros e pessoas que não se esperava serem vitimadas de COVID-19, mas o padrão é de aumento de segurança e de qualidade de vida (Lomborg, 2001; Rodrigues, 2014; Rosling, 2018; Pinker, 2018).

A evolução da esperança de vida à nascença, de cerca de quarenta anos no início do século XIX até aos atuais cerca de oitenta anos, tem momentos em que diminui (Van Zanden et al. 2014). Nota-se esse efeito na Alemanha e França durante as duas guerras mundiais que ocorreram no século XX (ver, e.g., Rodrigues, 2020). Mas a maior contribuição é a diminuição da mortalidade infantil e juvenil que tem evoluído quase linearmente desde o século XIX como se pode ver nesse trabalho. 
Ao longo da história e durante o século XX, ocorreram muitas epidemias (Martins et al. 1997; Aydon, 2010; Snowden, 2020). Também em Portugal (Morais, 2011, 2012a, 2012b), e estas eram tão comuns que poderiam ter menor destaque nas notícias (Almeida, 2014). Entretanto, Hayden (2004) refere muitos personagens conhecidas afetados pela sífilis e a história do tratamento para esta doença é bem conhecida (e.g. Liu et al, 2017; Vernon, 2019). Em Portugal, os números de prostitutas afetadas por esta doença foram anotados no hospital do desterro (Bastos, 2011). Estes relatórios mostram que as vias de transmissão eram múltiplas, muitas delas inocentes, contrariamente à ideia pública. Enquanto tuberculose e sífilis estão muito representadas na literatura e na arte, a gripe pneumónica aparece muito menos (Melo, 2009, p. 237).

Segunda refere Vieira (2015), "cada momento histórico teve as suas doenças emblemáticas." A lepra no mundo antigo; a peste negra na idade média; a cólera e a sífilis, a gripe pneumónica, a tuberculose, a partir do princípio do século XIX até meio do século XX; depois da segunda guerra mundial o cancro e a SIDA; e, atualmente, a COVID-19.

\section{Vidas salvas com a vacinação desde o seu início até hoje}

A história da vacinação é bem conhecida (pode ser consultada uma versão resumida em Plotkin, 2014). Somando uma estimativa grosseira de vidas salvas pela invenção de Jenner até ao século XX, com as estimativas mais precisas do século XX (Woodward, 2009; Science Heroes, 2021), dá cerca de mil milhões. Este valor, é quase igual ao da descoberta médica que é considerada mais importante em termos de vidas salvas: a descoberta dos grupos sanguíneos e da possibilidade de transfusões de sangue (Woodward, 2009; Science Heroes, 2021).

A primeira vacina para a COVID-19, aprovada para o público em geral, ficou disponível na Grã-Bretanha em 8 de dezembro de 2020 (BBC, 2020) e nos EUA a 11 de dezembro de 2020. Em Portugal a campanha de vacinação teve início a 27 de dezembro de 2020. Seria difícil resumir toda a informação disponibilizada sobre a vacinação para a COVID-19, mas esta pode ser encontrada nos sítios oficiais da Agência Europeia do Medicamento (Ema, 2021b) e na congénere dos EUA (FDA, 2021a). As revisões da literatura sobre o desenvolvimento de vacinas para a COVID-19 realizadas em dezembro de 2020 e publicadas no início de 2021 (e.g., Forni \& Montalvi, 2021; Sallam, 2021; Robinson et al., 2021) mostram simultaneamente que a ciência e as instituições reagiram prontamente e de forma eficaz, mas que as populações reagiram de forma conservadora e desconfiada e que nos países pobres a pandemia contribuiu para aumentar as desigualdades de acesso a vacinas. Em termos de vidas salvas, a Centro Europeu para o Controle e 
Prevenção de Doenças (ECDPC) e a WHO das Nações Unidas estimam em quase meio milhão de vidas salvas em cerca de um ano (ECDPC, 2021). As contribuições da química para a formulação das vacinas são em geral consideradas indiretas. A sua contribuição no desenvolvimento dos materiais utilizados, respetivamente, nas embalagens e excipiente é óbvia. No entanto, as contribuições da química podem ser consideradas mais diretas (veja-se a coleção de artigos coligidos pela American Chemical Society, 2022).

\section{Contribuições da química para a saúde pública}

As contribuições da química para a saúde pública podem ser organizadas em três categorias: alimentação, higiene e saúde. Uma lista de processos e medicamentos emblemáticos é apresentada na Tabela 1, assim como a data (ou datas aproximadas) da sua introdução, e ainda uma estimativa das vidas salvas (da morte precoce) quando essa estimativa está disponível (Woodward, 2009; Science Heroes, 2021); ver também (Rodrigues, 2016, 2020).

Table 1: Descobertas e invenções químicas que tiveram ou podem ter impacto no número de vidas salvas, assim como as suas datas aproximadas de introdução e número estimado de vidas salvas (ver texto). A verde, apresentam-se as descobertas ou invenções relacionadas com a alimentação, a azul as que estão relacionadas com a higiene e a cor de rosa as que são relacionadas com a saúde. Quando estas podem ter contribuído para várias categorias escolheu-se a que considerámos mais representativa.

\begin{tabular}{lll}
\hline Processo ou nome associado & Data aproximada & Estimativa de vidas salvas \\
\hline Processo Leblanc & 1791 & \\
\hline Vacina da varíola & 1794,1966 & 652 milhões \\
\hline Quinino & 1810,1818 & \\
\hline Processo Solvay & 1861 & \\
\hline Assepcia & 1862,1867 & 7 milhões \\
\hline Ácido acetilsalicílico & 1897,1950 & 177 milhões \\
\hline Cloro na água de consumo & $1895,1930,1941$ & 1100 milhões \\
\hline Grupos sanguíneos e transfusões & 1902,1913 & 2300 milhões \\
\hline Síntese da amónia & 1909 & \\
\hline Arsenamina & 1910 & 200 milhões \\
\hline CFC (clorofluorocarbonetos) & 1920 (banidos, 1989) \\
\hline Insulina & 1921 & \\
\hline Prontosil & 1932 & 203 milhões \\
\hline DDT & 1939 (banindo, 1970) 37 milhões \\
\hline Penicilina & 1943 & \\
\hline Dapsona & 1939,1945 \\
\hline Estreptomicina & 1948 \\
\hline
\end{tabular}




\begin{tabular}{lll}
\hline Processo ou nome associado & Data aproximada & Estimativa de vidas salvas \\
\hline Lindano & 1951 (banido, 2006) & \\
\hline Clorpromazina & 1952 & \\
\hline Desenho racional de fármacos & $1950-1960$ & \\
\hline Mercaptopurina & 1953 & \\
\hline Paracetamol & 1955 & \\
\hline Halotano & 1956 & \\
\hline Hexetidina & 1956 & \\
\hline Noretindrona & 1960 \\
\hline Diazepam & 1963 & \\
\hline Propanolol & 1964 & \\
\hline Rifampicina & 1968 \\
\hline Levodopa & 1970 \\
\hline Ciclosporina & 1971 \\
\hline Amoxicilina & 1971 \\
\hline Artemisinina & 1972 \\
\hline Ibuprofeno & 1974 \\
\hline Aciclovir & 1982 \\
\hline Permetrina & 1986 \\
\hline Fluoxetina & 1987 \\
\hline Diclofenac & 1988 \\
\hline Omeprazol & 1989 \\
\hline Sinvastatina & 1991 \\
\hline Paclitaxel & 1992 \\
\hline Lamivudina & 1995 \\
\hline Sildenafil & 1998 \\
\hline Dimeticona & 2006 \\
\hline Oseltamivir & 2012 \\
\hline Sofosbuvir & 2013 \\
\hline Flibanserina & & \\
\hline
\end{tabular}

\section{Contribuições da química para a alimentação}

A maior quantidade de vidas salvas é devida à descoberta da forma de obter adubos sintéticos. Estima-se que um terço da população mundial deva a sua vida a estes (Erisman et al. 2008). Entretanto, os adubos, muitas vezes em excesso, vão criar novos problemas, mas a ciência em geral, e a química em particular, estão a procurar resolvê-los. A agricultura de precisão e nova técnicas agrícolas, usando 
metodologias da química verde são alguns deles (este assunto foi mais desenvolvido por Rodrigues (2018).

Os clorofluorocarbonetos (CFC) estão associados à generalizações do uso da refrigeração doméstica, que contribuiu para salvar muitas vidas, evitando intoxicações alimentares e preservando nutrientes importantes. Desde os frigoríficos como luxo até à generalização destes equipamentos, deve-se muito aos CFC, que, entretanto, foram banidos e têm sido substituídos por moléculas ambientalmente mais seguras. Emsley (2010, 1-29) faz uma revisão das contribuições químicas para a alimentação, envolvendo outros aspetos não considerados aqui.

\section{Contribuições da química para a higiene}

Os processos Leblanc e Solvay estão associados aos sabões que, entretanto, deram origem a todo um conjunto de sabões, sabonetes, sabões líquidos e detergentes que estão ligados a atividades relacionadas com a higiene e combate a bactérias patogénicas. $O$ tratamento da água com cloro é também um grande passo que ainda não encontrou solução melhor, mas pode ser minimizada a adição deste elemento (que, nas concentrações usadas, não apresenta problemas além dos relacionados com o sabor e o cheiro). Com a filtração, a análise das águas e o controle e higiene rigorosos são evitados os maiores problemas e podem assim ser usadas menores quantidades de cloro (os valores que são usados atualmente em Portugal - 0,2 a 0,6 ppm de acordo com o Decreto-Lei n. ${ }^{\circ}$ 306/2007, de 27 de agosto - são em geral inferiores aos valores indicados pela literatura para a sua deteção pelo cheiro ou sabor, mas estes valores podem ser muito baixos, ver, e.g., Crider, 2018 e as referências aí presentes). Este elemento continua a ser necessário para garantir a esterilidade da água ao longo da rede. Os casos da bactéria Legionela que de vez em quando surgem, são em boa parte devidos a deficiências no processo de desinfeção. Também os tratamentos das águas incluem (ou podem incluir) a destruição de medicamentos e outros poluentes específicos.

O DDT, entretanto, banido na maioria dos países, contribuiu para o combate aos mosquitos que transmitiam doenças, em particular a malária, em muitas regiões, no Mediterrâneo por exemplo (Majori, 2012). Os inseticidas lindano (entretanto também banido) e a permetrina e os seus derivados têm contribuído para eliminar várias pragas de insetos que transmitiam doenças. Hoje em dia, não havendo problemas tão agudos como antigamente, é possível em muitos casos, com vários outros métodos e partindo dos resultados entretanto obtidos, preservar os insetos que eram parasitados pelas doenças, contribuindo para preservar a cadeia alimentar e o equilíbrio. Mas podemos fazer isto hoje (embora subsistam problemas, nomeadamente de biodiversidade) porque muitos problemas já foram 
resolvidos antes. A dimeticona permite eliminar parasitas, em particular piolhos, de forma mais segura, envolvendo-os no polímero que forma. O número de vidas salvas associado a este composto pode ser muito pequeno, mas esta molécula é relevante como paradigma dos desenvolvimentos químicos que permitem eliminar parasitas de formas menos tóxicas.

Finalmente, a hexetidina é apresentada como paradigma de um desinfetante externo. Há atualmente algumas questões sobre o seu uso (ver, e.g., Goodfellow, 2020), mas é preciso lembrar que sua invenção permitiu níveis de desinfeção que só podiam ser obtidos com desinfetantes mais perigosos (como o fenol ou a lixívia).

\section{Contribuições da química para a saúde}

As contribuições químicas para a saúde foram analisadas por vários autores, dos quais se destaca Emsley (2010, pp. 54-76). Os antibióticos, em particular as penicilinas, são muito conhecidos, mas o número de vidas salvas devido a eles é relativamente pequeno comparado com os dos adubos sintéticos e vacinas. A história da descoberta e desenvolvimentos da penicilina é contada em detalhe em (Rodrigues, 2020). Far-se-à aqui um resumo. Alexander Fleming (1881-1955) descobriu em 1928 um fungo combatia bactérias, mas não conseguiu isolar o composto responsável. Num grande projeto, durante a Segunda Guerra Mundial, Howard Florey (1888-1968), Ernst Chain (1907-1979) e as suas equipas conseguiram isolar a penicilina, mas não a produzir em grande quantidade. Isso foi feito nos EUA, e, em 1943, já havia penicilina disponível para os soldados. Fleming, Florey e Chain ganharam o prémio Nobel da Medicina em 1945. Para estabelecer a estrutura molecular da penicilina contribuíram Dorothy Hodgkin (1910-1994) e vários outros cientistas. O projeto chegou a envolver milhares de cientistas, mas estes não conseguiram obter penicilina sintética. Isso só foi conseguido em 1957 por John C. Sheehan (1915-1992) e a sua equipa, abrindo caminho para as penicilinas semi-sintéticas como a amoxicilina. Foram também importantes a descoberta de outros antibióticos como sejam as cicloesporinas e as tetraciclinas, entre outros, pois as bactérias vão ganhando resistências a estas moléculas. Antes dos antibióticos, só no século XX se obtiveram alguns resultados com as sulfamidas, de que o prontosil é exemplo. Infelizmente, os efeitos secundários destes compostos eram muito mais relevantes do que os dos antibióticos.

Um medicamento histórico para a malária é o conhecido quinino (uma revisão e métodos propostos para encontrar novos medicamentos para esta doença pode ser encontrada em Fidock et al., 2004). É difícil fazer uma estimativa de vidas salvas desde a sua descoberta até este ter perdido eficácia, e, depois, com a introdução dos medicamentos que se lhe seguiram, tanto sintéticos como naturais. 
O ácido acetilsalicílico, tem um papel múltiplo. Sendo inicialmente um analgésico, como o paracetamol, o ibuprofeno ou diclofenac, cujas contribuições para salvar vidas são difíceis de avaliar. O ácido acetilsalicílico contribui para o número de vidas salvas essencialmente pelas suas características anti-trombóticas. A arsenamina foi o primeiro medicamento não mercúrico para a sífilis. A insulina é uma hormona necessária para a diabetes tipo I. A dapsona é o primeiro medicamento para a lepra, doença que até meio dos anos quarenta do século XX não tinha cura. A estreptomicina é um dos primeiros medicamentos eficazes para a tuberculose, doença para a qual as penicilinas não eram eficazes. No entanto, só com a rifampicina, que vai ficar disponível pelos anos sessenta do século XX, se irá combater esta doença de forma eficaz.

O desenho e descoberta racional de fármacos irão começar a ser feitos pelos anos 1950 e levará ao desenvolvimento de muitos medicamentos. Um destes é a mercaptopurina, eficaz para alguns tipos de leucemia, ou o paclitaxel para outros tipos de cancro, obtido este último pesquisando de forma sistemática. Atualmente, uma boa parte dos medicamentos não têm origens naturais, nem são bioinspirados, sendo obtidos pesquisando sistematicamente a atividade biológica e a seletividade de moléculas desenhadas usando relações atividade-estrutura ou de outras formas. Uma análise que procura evidenciar a natureza como origem de medicamentos, mostra que, das entidades farmacológicas aprovadas entre 1981 a 2014, cerca de metade não tem desenvolvimento baseado em compostos naturais (Newman \& Cragg, 2016). É também difícil avaliar as contribuições de moléculas usadas em anestesia como o halotano, mas as suas contribuições são indiretas. A sinvastatina é um exemplo das estatinas, grupo de moléculas que baixam a concentração de colesterol no organismo. São importantes, pois foi descoberto nos anos 1960 que havia uma relação entre excesso de colesterol e ataque cardíaco. Atualmente, as condições da sua utilização têm sido revistas, nomeadamente com valores mais altos de colesterol aceites e influência de outros riscos (ver, e.g., consenso da Sociedade Europeia de Arteriosclerose (EAS, 2022) e as referências aí presentes). O propanolol é um paradigma de um medicamento para baixar a tensão arterial, que aumenta o risco de problemas cardíacos. O aciclovir é o primeiro antiviral, o qual abriu espaço para medicamentos como a lemivudina, o oseltamivir e medicamentos que estão a ser desenvolvidos para a gripe e os coronavirus. O oseltamivir é paradigmático por ser o primeiro medicamento desenvolvido de forma computacional. Poder-se-ia pensar que o omeprazol não salvou vidas, mas o excesso de acidez no estômago pode levar a úlceras e estas a doenças mais complicadas. O levodopa é o primeiro medicamento para o Parkinson e terá grande impacto na qualidade de vida destes doentes. 
É difícil avaliar o impacto dos medicamentos psicotrópicos, mas pode-se imaginar que o número de suicídios que foram evitados é muito maior do que os provocados e do que as intoxicações que tiveram lugar. Uma coisa é certa: o número de internamentos diminuiu (Meyer \& Quenzer, 2005, p. 445). A clorpromazina (o primeiro anti-psicótico), o diazepam (a primeira benzodizepina, calmantes que substituíram os barbitúricos, moléculas muito mais perigosas) e a fluoxetina (modificar do humor, o primeiro recaptador seletivo da serotonina) têm tido um impacto social enorme (Rodrigues, 2019, aqui podem ser encontradas referências sobre o desenvolvimento de vários dos medicamentos psicotrópicos). É também quase impossível avaliar numericamente, mas os efeitos são bem visíveis, qual tem sido o papel dos contracetivos orais, que tiveram a sua origem com a noretindrona. Para além do impacto social, estes têm também efeitos na qualidade de vida e segurança. São também difíceis de avaliar os efeitos de medicamentos como o sildenafil (conhecido como viagra) ou mais recentemente da flibanserina que ficou conhecia como o "viagra feminino".

Na lista da Tabela 1 estão outros medicamentos que, embora se espera tenham tido um impacto relativamente pequeno, como o sofosbuir, para a hepatice $\mathrm{C}$, tiverem um grande impacto na descoberta de cura para esta doença.

Todos os anos são aprovados cerca de três a cinco dezenas de novas moléculas pela Food and Drug Administration dos EUA (FDA, 2021c), sendo um número semelhante aprovado pela European Medicines Agency na UE. Trata-se de um processo cada mais vez mais complicado pois as novas entidades moleculares têm de demonstrar não ser tóxicas, que são mais eficazes do que as que já existem (caso sejam propostas para a mesma doença), sendo cada vez mais complexas as doenças para as quais ainda não há tratamento (para mais detalhes ver, e.g., RODRIGUES, 2019). Mas com a ciência em geral, e a química em particular, podemos continuar a manter confiança no futuro.

\section{Limitações da presente revisão}

Há duas estimativas presentes no site (Science Heroes, 2021) que foram usadas ao longo to trabalho e merecem alguma reserva. A vidas salvas devido ao começo do uso das vacinas e aos adubos sintéticos. Na versão anterior do site estimavam as vidas salvas devido à invenção da vacina da varíola por Edward Jenner em 530 milhões. Esse número é muito difícil de estimar. De qualquer forma, a esse número somei o valor das vidas salvas devido à irradicação desta doença (122 milhões), o que dá o número de 652 milhões. Se somarmos todas estimativas de vidas salvas devido às vacinas, dá cerca de 1000 milhões, um 
número quase igual ao da descoberta dos grupos sanguíneos que estes autores apresentam. A estimativa que estes fazem para vidas salvas devido às vacinas da COVID-19 é de cerca um milhão, quando as estimativas mais recentes apontam para metade (ECDPC, 2021). O outro número problemático é o das vidas salvas devido aos adubos sintéticos. Na versão anterior estavam 2720 milhões de vidas, mas na versão atual estão 2300 milhões de vidas. Trata-se de um número difícil de estimar, mas tomando os valores de (Erisman et al. 2008), autores que sugerem entre 30 a $40 \%$ da humanidade, obtemos um número entre 2300 milhões e 3000 milhões de pessoas.

Os adubos sintéticos e os CFC são atualmente problemáticos, embora as suas contribuições tenham sido muito importantes para a melhoria de bem-estar que temos hoje (Rosling, 2018; 2021, Pinker, 2018). Outros problemas são a poluição (e.g. Bell, Zanobetti \& Dominici, 2014) e ameaças muito graves como o aumento do aquecimento global e a diminuição de biodiversidade. Para todas estas questões a química pode contribuir (Rodrigues, 2018), nomeadamente através de processos mais sustentáveis e circulares e produção de alimentos sintéticos, libertando espaços de produção agrícola, mas ainda há bastante incerteza sobre as soluções.

\section{Conclusões}

Fez-se neste trabalho uma revisão das invenções, descobertas e desenvolvimentos ligados à química que têm contribuído para a melhoria da saúde pública, nomeadamente para salvar vidas e contribuir para o aumento da esperança de vida e bem-estar e segurança. Estes são essencialmente de três tipos: segurança alimentar, higiene e o desenvolvimento de tratamentos para doenças, que em muitos casos quase desapareceram. Naturalmente esta revisão não poderia ser exaustiva, mas procura dar uma visão geral das contribuições da química para estas áreas da atividade humana. Embora as aplicações da química possam parecer só envolver a descoberta de novos medicamentos e curas para doenças, trata-se de uma contribuição muito mais vasta que deve ser conhecida e reconhecida. Não deve ficar invisível por os materiais e processos que parecem tão comuns que nem se pensa neles, nem deve ser obscurecida pelos problemas que vão surgindo.

Agradecimentos:

O Centro de Química de Coimbra (CQC) é apoiado pela Fundação para a Ciência e a Tecnologia (Projetos UIDB/00313/2020 e UIDP/00313/2020). 


\section{Referências}

American Chemical Society (2022). Chemistry in Coronavirus Research: A Free to Read Collection from the American Chemical Society. https://pubs.acs.org/page/vi/chemistry_coronavirus_research

Almeida, M. A. P. (2014). As epidemias nas notícias em Portugal: cólera, peste, tifo, gripe e varíola, 1854-1918, História, Ciências, Saúde-Manguinhos, 21, 687-708. http://dx.doi.org/10.1590/S0104-59702014000200012

Ansah, J. P., Matchar, D. B., Shao Wei, S. L., Low J. G., Pourghaderi, A. R., Siddiqui, F. j., et al. (2021). The effectiveness of public health interventions against COVID-19: Lessons from the Singapore experience, PLoS ONE 16, e0248742. https://doi.org/10.1371/journal.pone.0248742

Aydon, C. (2010). Breve história da Humanidade. Lisboa: Gradiva.

BASTOS, C. (org.) (2011). Clínica, arte e sociedade: a sifilis no Hospital do Desterro e na saúde pública. Imprensa de Ciências Sociais, Lisboa.

BBC (2020, dezembro 8). First person to get COVID vaccine is happy to inspire others. https://bbc.in/3HXBrxG

Bell, M. L., Zanobetti, A., \& Dominici, F. (2014). Who is More Affected by Ozone Pollution? A Systematic Review and Meta-Analysis, American Journal of Epidemiology. 180(1), 15-28. https://doi.org/10.1093/aje/kwu115

Cinar, F., Ekinci, G. (2022). Investigation of the Effect of Comorbidity on Mortality in Patients with COVID-19: A Systematic Review and MetaAnalysis. Biointerface Research in Applied Chemistry, 12(4), 5579-5590. https://doi.org/10.33263/BRIAC124.55795590

CHRISTENSEN, Jen (2021, novembro 25). Reinfection from COVID-19 is rare, severe disease is even rarer, a study of people in Qatar finds. CNN. https://cnn.it/3BmWHdJ

Crider, Y., Sultana, S., Unicomb, L., Davis, J., Luby, S. P., \& Pickering, A. J. (2018). Can you taste it? Taste detection and acceptability thresholds for chlorine residual in drinking water in Dhaka, Bangladesh. Science of the Total Environment, 613-614, 840-846. https://doi.org/10.1016/j.scitotenv.2017.09.135

Diário de Lisboa: Folha oficial do Governo Portuguez (1862, fevereiro 22). Diário de Lisboa, pp. 557-562.

ECDPC (2021, novembro 25). WHO/ECDC: Nearly half a million lives saved by COVID-19 vaccination in less than a year. European Centre for Disease Prevention and Control. https://bit.ly/3HTkuUR

EMA (2021a). Public-health advice during COVID-19 pandemic. European Medicines Agency. https://bit.ly/34QKOjT

EMA (2021b). COVID-19 vaccines. European Medicines Agency. https://bit.ly/3oQBq6S

EMA (2021c). EMA issues advice on use of Paxlovid (PF-07321332 and ritonavir) for the treatment of COVID-19: rolling review starts in parallel. European Medicines Agency. https://bit.ly/3oRtbHN 
EMSLEY, John (2010). A healthy, Wealthy, Sustainable World. RSC Publishing.

Erisman, J., Sutton, M., Galloway, J., Zbigniew K., \& Wilfried W. (2008). How a century of ammonia synthesis changed the world. Nature Geosci, 1, 636-639. https://doi.org/10.1038/ngeo325

EAS (2022). EAS Consensus panel answers the questions on statin safety. European Atherosclerosis Society. https://www.eas-society.org/page/statin_safety

FDA (2021a). COVID-19 Vaccines. U.S. Food and Drug Administration. https://bit.ly/3H2EXWh

FDA (2021b). Coronavirus (COVID-19) Update: FDA Authorizes First Oral Antiviral for Treatment of COVID-19. U.S. Food and Drug Administration. https://bit.ly/3rOBaY4

FDA (2021c). Novel Drug Approvals for 2021. U.S. Food and Drug Administration. https://bit.ly/3I2KbSQ

Felix, C. B., Ubando, A. T., Chen, W. H., Goodarzi, V., Ashokkumar, V. (2022). COVID-19 and industrial waste mitigation via thermochemical technologies towards a circular economy: A state-of-the-art review. Journal of Hazardous Materials, 423B, 127215. https://doi.org/10.1016/j.jhazmat.2021.127215

Fidock, D. A., Rosenthal, P. J., Croft, S. L., Brun, R., \& Nwaka, S. (2004). Antimalarial drug discovery: efficacy models for compound screening. Nature reviews Drug discovery, 3(6), 509-520. https://doi.org/10.1038/nrd1416

Forni, G., \& Mantovani, A. (2021). COVID-19 vaccines: where we stand and challenges ahead. on behalf of the COVID-19 Commission of Accademia Nazionale dei Lincei, Rome. Cell Death Differ, 28, 626-639. https://doi.org/10.1038/s41418-020-00720-9

Goodfellow, K. (2020, abril 1). Chlorhexidine - the good, the bad, and the ugly. $R D H$. https://bit.ly/3GIW9Qk

Hayden, D. (2004). Pox: genius, madness, and the mysteries of syphilis. Perseus Books.

Keinan, E. (2013). Gloomy Forecast for the Prophets of Apocalypse and Bright Forecast for Chemists, Angewandte Chemie, 52(10), 2667-2672. https://doi.org/10.1002/anie.201209383

Kumar, A., Singh, P., Raizada, P., \& Hussain, C. M. (2022). Impact of COVID-19 on greenhouse gases emissions: A critical review, Sci. Total Env. 806, 150349. https://doi.org/10.1016/j.scitotenv.2021.150349

LANDES, David S. (2001). A riqueza e a pobreza das nações. Gradiva.

LASZLO, Pierre (1996). A nova química. Edições Piaget.

Li, M. T., Song, G., Liu, R. P., Huang, X., \& Liu, H. (2022). Inactivation and risk control of pathogenic microorganisms in municipal sludge treatment: A review. Frontiers of Environmental Science \& Engineering, 16, 70. https://doi.org/10.1007/s11783-021-1504-5 
Liu H-y., Han Y., Chen X-s., Bai L., Guo S-p., Li L., Wu, P. \& Yin, Y-p. (2017) Comparison of efficacy of treatments for early syphilis: A systematic review and network meta-analysis of randomized controlled trials and observational studies. PLoS ONE 12(6): e0180001. https://doi.org/10.1371/journal.pone.0180001

LOMBORG, Bjorn (2001). The Skeptical Environmentalist: Measuring the Real State of the World. New York: Cambridge University Press.

Majori, G. (2012). Short History of Malaria and its Eradication in Italy Mediterranean Journal of Hematology and Infectious Diseases, 4(1), p. e2012016. https://doi.org/10.4084/MJHID.2012.016

Martins, R. A., Martins, L. A. P., Ferreira, R. R., \& Toledo, M. C. F. (1997). Contágio: história da prevenção das doenças transmissiveis. São Paulo: Moderna. http://www.ghtc.usp.br/Contagio

Mattoso, J. (2015). Identificação de um país. Temas e Debates.

Mattoso, J. (2020). A história Contemplativa. Temas e Debates.

Melo, D. (2009). A gripe pneumónica no Portugal Republicano: o olhar dos escritores e fotógrafos. Em J. Sobral, L. Lima, P. Castro, P. S. Sousa (Eds). A Pandemia esquecida - olhares comparados sobre a pneumónica 1918-1919. Lisboa: Imprensa de Ciências Sociais.

Meyer, J. S., Quenzer, Linda F. (2005). Psychopharmacology: drugs, the brain, and behavior. Sunderland: Sinauer.

Moabelo, K. L., Martin, D. R., Fadaka, A. O., Sibuyi, N. R. S., Meyer, M., \& Madiehe, A. M. (2021). Nanotechnology-Based Strategies for Effective and Rapid Detection of SARS-CoV-2. Materials, 14(24), 7851. https://doi.org/10.3390/ma14247851

Morais, J. A. D. (2011). Surtos epidémicos ocorridos em Portugal na primeira metade do século XX: abordagem histórico-epidemiológica. I - Peste bubónica. Medicina Interna, 18, 259-266. https://bit.ly/3uRx0Ri

Morais, J. A. D. (2012a). Surtos epidémicos ocorridos em Portugal na primeira metade do século XX: abordagem histórico-epidemiológica. II - Varíola, Medicina Interna, 19, 43-50. https://bit.ly/3HPYg6n

Morais, J. A. D. (2012b). Surtos epidémicos ocorridos em Portugal na primeira metade do século XX: abordagem histórico-epidemiológica. III - Gripe pneumónica, Medicina Interna, 19, 97-104. https://bit.ly/3LwCg2t

Newman, D. J., \& Cragg, G. M. (2016). Natural Products as Sources of New Drugs from 1981 to 2014, Journal of Natural Products, 79(3), 629-661. https://doi.org/10.1021/acs.jnatprod.5b01055

Ritchie, H., Mathieu, E., Rodés-Guirao, L., Appel, C., Giattino, C., Ortiz-Ospina, E., Hasell, J., Macdonald, B., Beltekian, D., \& Roser, M. (2021). Coronavirus Pandemic (COVID-19). OurWorldInData.org. https://bit.ly/3sJGCdQ

Pinker, S. (2018). Enlightment Now: The Case for reason. Science, Humanism, and Progress. Great Britain: Allen Lane. 
Plotkin, S. (2014). History of vaccination. PNAS, Proceedings of the National Academy of Sciences, 111(34), 12283-12287. https://doi.org/10.1073/pnas.1400472111

Porto, V. A., \& Porto, R. S. (2022). In silico Studies of Novel Synthetic Compounds as Potential Drugs to Inhibit Coronavirus (SARS-CoV-2): A Systematic Review, Biointerface Research in Applied Chemistry, 12(4), 4293-4306. https://doi.org/10.33263/BRIAC124.42934306

Robinson, E., Jones, A., Lesser, I., \& Daly, M. (2021). International estimates of intended uptake and refusal of COVID-19 vaccines: A rapid systematic review and meta-analysis of large nationally representative samples. Vaccine, 39(15), 2024-2034. https://doi.org/10.1016/j.vaccine.2021.02.005

Rodrigues, S. (2014). Jardins de cristais - Química e literatura. Lisboa: Gradiva, 2014.

Rodrigues, S. P. J. (2016a). Que Química! Entre o fascínio com o pessimismo e a hesitação perante o optimismo. Química - Bul. Soc. Port. Quim., 140, 27-35.

Rodrigues, S. P. J. (2016b). Mal-entendidos, preconceitos e mitos sobre química na sociedade contemporânea. In Oliveira, M., Santos, O., Carvalho, N., Lameiras, E., Castro, J. (Coord.) Atas do II Congresso Internacional Educação, Ambiente e Desenvolvimento. (pp. 317-330). Leiria: OIKOS. Ebook ISBN 978-989-99054-2-9.

Rodrigues, S. P. J. (2018). Acerca das contribuições da química para os objectivos de desenvolvimento sustentável das Nações Unidas. In Santos, O., Oliveira, M., Carvalho, N. (Coord.) Atas do III Congresso Internacional Educação, Ambiente e Desenvolvimento. (pp. 338-351). Leiria: OIKOS. E-book SBN 978-989-99054-4-3.

Rodrigues, Sérgio P. J. (2019). Moléculas psicotrópicas e literatura: uma visão química. Em História interdisciplinar da loucura, psiquiatria e saúde mental $I X$. M. L. Pereira, J. R. Pita, (Coords.) Coimbra: Sociedade de História Interdisciplinar da Saúde / Centro de Estudos Interdisciplinares do Século XX da Universidade de Coimbra - CEIS20 (Grupo de História e Sociologia da Ciência e da Tecnologia).

Rodrigues, Sérgio P. J. (2020). A Química ao serviço da vida e prisioneira da guerra, em I. Abrantes, P. M. Callapez, G. P. Correia, E. Gomes, B. Lopes, F. C. Lopes, E. Pires, \& A. Rola (Eds.), Uma visão holística da Terra e do Espaço nas suas vertentes naturais e humanas. Homenagem à Professora Celeste Romualdo Gomes. Coimbra: CITEUC, pp. 229-242.

Rosling, H., Rosling, O., Ronnlund, A. R. (2018). Factfulness. Lisboa: Círculo de Leitores.

Rosling, H., \& Härgesteam, F. (2021). Como aprendi a compreeder o mundo. Lisboa: Círculo de Leitores.

Sallam, M. (2021). COVID-19 Vaccine Hesitancy Worldwide: A Concise Systematic Review of Vaccine Acceptance Rates. Vaccines, 9(2), 160. https://doi.org/10.3390/vaccines 9020160 
Science Heroes (2021). Who saved the most lives in history?! scienceheroes.com. https://www.scienceheroes.com/

Snowden, F. M. (2020). Epidemias e sociedade: da Peste Negra ao presente. Edições 70.

Van Zanden, J. L., Baten, J., d'Ercole, M. M., Rijpma, A., Smith, C., \& Timmer M. (eds.). (2014). How Was Life?: Global Well-being since 1820. OECD Publishing.

Vernon, G. (2019). Syphilis and Salvarsan. British Journal of General Practice, 69(682), 246. https://doi.org/10.3399/bjgp19X702533

Vieira, I. C. (2015). Conhecer, tratar e combater a "peste branca". Lisboa: Edições Afrontamento.

Wang J., Kaperak C., Sato T., \& Sakuraba, A. (2021). COVID-19 reinfection: a rapid systematic review of case reports and case series. Journal of Investigative Medicine, 69, 1253-1255. http://dx.doi.org/10.1136/jim-2021-001853

WHO (2021a) Considerations for implementing and adjusting public health and social measures in the context of COVID-19. World Health Organization. https://bit.ly/33kek0X

WHO (2021b). WHO Coronavirus (COVID-19) Dashboard. World Health Organization. https://COVID19.who.int/

Woodward, B., Shurkin, J. N., \& Gordon, D. (2009). Scientists greater than Einstein: the biggest lifesavers of the twentieth century. Fesno: Quill Driver Books.

WORLDOMETER (2021). COVID-19 Coronavirus Pandemic. Worldometer. https:/www.worldometers.info/coronavirus/ 